\title{
Estimación de la biomasa aérea total y por componente estructural en especies arbóreas de Lima, Perú
}

\author{
Estimation of the total aboveground biomass and by structural components in tree \\ species from Lima, Peru
}

Karin Begazo Curie ${ }^{1, \star}$ y Jorge Jave Nakayo ${ }^{2}$

\begin{abstract}
Resumen
La presente investigación tuvo como objetivos estimar la biomasa aérea y por componente estructural del árbol, realizar una comparación intraespecífica e interespecífica de estas variables, así como también generar modelos alométricos de biomasa aérea total para cada especie. A través de ensayos destructivos, se evaluaron 44 individuos en total: 15 individuos de la especie Tecoma stans (L.) Juss. ex Kunth var. stans, 15 individuos para Grevillea robusta A. Cunn. ex R. Br, y 14 individuos para Schinus molle L. Para la determinación de la biomasa aérea y la selección de los modelos alométricos se siguió la metodología sugerida por Picard et al. (2012) y Segura y Andrade (2008). Los resultados de la biomasa aérea fueron de $43.18 \mathrm{~kg} /$ árbol para T. stans, $68.49 \mathrm{~kg} /$ árbol para G. robusta y $32.94 \mathrm{~kg} /$ árbol para $S$. molle, no encontrándose diferencias estadísticamente significativas entre las tres especies. Sin embargo, cuando se comparó la cantidad de biomasa por componente estructural, es el fuste el componente que difiere significativamente entre las tres especies, siendo la especie G. robusta la que presenta la mayor cantidad de biomasa en este componente. Se comparó la cantidad de biomasa por componentes dentro de la misma especie, y se encontró que existen diferencias significativas. En el caso de $T$. stans y S. molle, el componente que difiere son las hojas. En el caso de G. robusta, el componente que difiere en este caso es el fuste. Para la selección de los modelos se utilizaron como variables explicativas al diámetro y la altura total. Se seleccionaron modelos alométricos, de mayor ajuste considerando el Error Estándar de la Estimación y el Índice de Furnival. Los modelos alométricos seleccionados fueron: $\operatorname{Ln}(B T)=-2.9409+2.6925$ $\times \operatorname{Ln}($ dap) para $T$. stans, $\operatorname{Ln}(B T)=-2.0082+2.3293 \times \operatorname{Ln}($ dap) para G. robusta y $\operatorname{Ln}(B T)$ $=-3.3201+1.4834 \times \operatorname{Ln}($ dap $\times$ ht $)$ para S. molle.
\end{abstract}

Palabras clave: Biomasa sobre el suelo, modelos alométricos, Tecoma stans, Schinus molle, Grevillea robusta.

\footnotetext{
${ }^{1}$ Departamento de Industrias Forestales, Facultad de Ciencias Forestales, Universidad Nacional Agraria La Molina (UNALM), Av. La Molina s/n, La Molina, Lima, Perú.

${ }^{2}$ Unidad de Posgrado de la Facultad de Ingeniería Geológica, Minera, Metalúrgica y Geográfica, Universidad Nacional Mayor de San Marcos (UNMSNM), Av. Venezuela 3400, Lima, Perú.

* Autor de Correspondencia: kbegazo@lamolina.edu.pe
} 


\begin{abstract}
The aim of the research was to estimate the total aboveground biomass and by structural component, to make intraspecific and interspecific comparisons among this variables, and to generate allometric models for total aboveground biomass. Through destructive tree sampling, 44 trees were evaluated: 15 for Tecoma stans (L.) Juss. ex Kunth var. stans, 15 from Grevillea robusta A. Cunn. ex R. Br, y 14 individuals for Schinus molle L. In order to determine the total aboveground biomass and to select the allometric model, it was followed the procedure suggested by Picard et al. (2012) and Segura and Andrade (2008). The results of the biomass estimation were $43.18 \mathrm{~kg} /$ tree for T. stans, $68.49 \mathrm{~kg} /$ tree for G. robusta y $32.94 \mathrm{~kg} /$ tree for S. molle. No statistical differences were found for these results. However, when compared the amount of aboveground biomass for components between species, it was found significant differences. The trunk of G. robusta was the component that differs with the most amount of aboveground biomass. In addition, it was compared the amount of aboveground biomass among tree component inside the specie. The results obtained shows that there were significant differences among structural components inside the specie. T. stans and S. molle are the species whose leaves had the low amount of biomass. In the case of G. robusta, the trunk is the component that differs and presents the larger amount of aboveground biomass among its components. Allometric models were generated for the tree species. The selection of the adjusted model considered the lowest values for the Root Square Mean Error and the Furnival Index. The selected models were: $\operatorname{Ln}(B T)=-2.9409+2.6925 \times \operatorname{Ln}($ dap $)$ for T. stans, $\operatorname{Ln}(B T)$ $=-2.0082+2.3293 \times \operatorname{Ln}($ dap $)$ for $G$. robusta and $\operatorname{Ln}(B T)=-3.3201+1.4834 \times \operatorname{Ln}($ dap $\times$ ht) for S. molle.
\end{abstract}

Key words: Aboveground biomass, allometric models, Tecoma stans, Schinus molle, Grevillea robusta.

\section{Introducción}

El arbolado y los bosques urbanos juegan un papel importante en las ciudades, pues contribuyen a mitigar los niveles de $\mathrm{CO}_{2}$ a través del almacenamiento y secuestro de carbono, así como también proveen múltiples beneficios a la comunidad como la mejora en la calidad del aire (captación de material particulado) y del agua, espacios para la recreación, ocio y relajación, reducción de la demanda de energía al generar un microclima, genera un valor emocional y estético para los residentes, provee de hábitat para fauna local, entre otros (Ferrini y Fini 2011). Sin embargo, no son valorados en su real dimensión, ya que los servicios ecosistémicos que presentan, no son bien entendidos ni cuantificados, siendo escasa la información documentada sobre esta temática en escenarios urbanos.

Los árboles ubicados en áreas urbanas experimentan mayor estrés (contaminación, podas, plagas, daños estructurales, entre otros) que los ubicados en ecosistemas naturales, por ello los valores reportados de biomasa sobre el suelo y almacenamiento y secuestro de carbono difieren entre sí cuando se trabaja en su cuantificación utilizando metodologías desarrolladas para bosques naturales (Nowak y Crane 2002). Es por ello, que se requiere investigación específica para el arbolado urbano, además de estrategias y políticas especiales para el desarrollo de estas áreas así como para futuros planes de incremento de espacios verdes en las ciudades.

Picard et al. (2012), señalan que existe una relación estadística entre las diferentes medidas de un individuo, esta relación se deriva del desarrollo ontogénico de los individuos que es la misma para todos, salvo la variabilidad asociada a la historia personal de cada uno. Así, las proporciones entre altura y diámetro, entre tamaño de la copa del árbol y el diámetro, entre la biomasa y el diámetro, obedecen a una regla que es la misma para todos los árboles que se desarrollan bajo las mismas condiciones, desde el más pequeño al más grande (King 1996; Archibald y Bond 2003; Bohlman y O’Brien 2006; 
Dietze et al. 2008, citado por Picard et al. 2012). Se trata del principio básico de la alometría que permite predecir una medida de un árbol (lo típico es su biomasa) en función de otra medida (por ejemplo, el diámetro). Por lo tanto, pesar los árboles para medir la biomasa forma parte fundamental del proceso de elaboración de ecuaciones alométricas.

Los estudios sobre cuantificación de biomasa y almacenamiento de carbono no son solo importantes para comprender la dinámica del ecosistema forestal urbano sino para entender las áreas verdes, no solo como un lugar de esparcimiento, sino como un recurso que puede generar valor ambiental y económico a la comunidad. Asimismo, los proyectos de captura de carbono generados en el marco del $\mathrm{Me}$ canismo de Desarrollo Limpio del Protocolo de Kioto, Mercados Voluntarios y los mecanismos de Reducción de Emisiones por Deforestación y Degradación Evitadas (REDD), requieren de información confiable para la negociación de los proyectos de captura o reducción de emisiones de los GEI.

En este contexto, el objetivo del presente trabajo fue determinar la biomasa aérea total y por componente estructural de especies forestales, realizar comparaciones dentro de la especie y entre especies, y a partir de dicha información generar ecuaciones alométricas de la biomasa aérea total para tres especies forestales presentes en Lima: Tecoma stans (L.) Juss. ex Kunth var. stans (Bignoniaceae), Grevillea robusta A. Cunn ex R. Br. (Proteaceae) y Schinus molle L. (Anacardiaceae). Dicha información será de utilidad en la selección de especies para una planificación de áreas verdes basados en criterios ambientales.

\section{Materiales y Métodos}

\section{Localización del área de estudio}

El presente estudio se realizó en el "Bosque simulado y Plantación forestal" del Vivero Forestal de la Facultad de Ciencias Forestales (FCF) de la Universidad Nacional Agraria La Molina, ubicado en el distrito de La Molina, en el Departamento de Lima (Perú). Geográficamente se ubica a $12^{\circ} 05^{\prime} 06^{\prime \prime}$ de Latitud Sur y $76^{\circ} 75^{\prime} 00^{\prime \prime}$ de Longitud Oeste, y a una altitud de 238 m.s.n.m. (Chirinos 2005).

El "Bosque simulado y Plantación forestal", posee una extensión de 6 ha, en la cual se encuentra una plantación forestal instalada desde el año 1998, y en la que predominan las especies: Schinus molle, Tecoma stans, Grevillea robusta, Eucaliptus globulus Labill, Ficus benjamina L. y Casuarina cunninghamiana Miq. El distanciamiento entre plantas es de $2 \times 2 \mathrm{~m}$, con corredores de $4 \mathrm{~m}$ entre hileras que permite el paso de luz. El manejo silvicultural (podas y raleos) es cada 3 años. El riego se realiza por inundación cada 15 días.

\section{Características del área de estudio}

El clima del distrito de La Molina es por lo general templado, con temperaturas mínimas mensuales promedio de $16.2{ }^{\circ} \mathrm{C}$ y máximas de $22.5^{\circ} \mathrm{C}$, una humedad relativa promedio anual de $81 \%$ y una precipitación total anual de 10 mm (UNALM 2018).

El área de estudio se caracteriza por tener suelos profundos, de buen drenaje, permeabilidad moderada y textura media a ligeramente gruesa. El suelo se encuentra dentro de los fluvisol eutrico; de origen aluvial, presentando una textura franco arenosa con un $\mathrm{pH}$ neutro y baja cantidad de materia orgánica (1\% a $45 \%$ $\mathrm{kg} \mathrm{N}$ mineral) y una C.I.C de 6.4. En cuanto a la disponibilidad de nutrientes el suelo presentó un nivel medio de fósforo $(92.74 \mathrm{~kg} / \mathrm{ha}$ $\mathrm{P}_{2} \mathrm{O}_{5}$ ) y potasio $\left(87.21 \mathrm{~kg} / \mathrm{ha} \mathrm{K}_{2} 0\right.$ ) (Chirinos 2005).

\section{Descripción de las especies estudiadas}

Tecoma stans ("huaranguay"): Árbol siempreverde a semicaducifolio de porte pequeño a mediano. Es una especie con velocidad de recimiento rápido que puede alcanzar los $8 \mathrm{~m}$ de altura. Tiene una copa globosa e irregular con un diámetro de 2 a $4 \mathrm{~m}$. Presenta ramas largas y delgadas. Las hojas son compuestas y las flores son numerosas con forma de campánulas de color amarillo. El tronco es delgado y suele seccionarse en ramificaciones, Las raíces 
son de crecimiento medianamente profundo y fasciculado (Bulnes et al. 2017).

Schinus molle ("molle peruano"): Árbol siempreverde de porte mediano, con velocidad de crecimiento moderado a rápido, que puede alcanzar los $8 \mathrm{~m}$ de altura. La copa es de forma irregular compuesta de ramas con frondas colgantes, de 6 a $9 \mathrm{~m}$ de diámetro. Las hojas son compuestas y las flores son muy pequeñas e imperceptibles. Los frutos están dispuestos en racimos. El tronco es de sinuoso a irregular y las raíces son de profundidad media a fasciculada (Bulnes et al. 2017).

Grevillea robusta (“grevilea”): Árbol siempreverde a semicaducifolio de porte alto, con velocidad de crecimiento moderado a rápido que puede alcanzar los $20 \mathrm{~m}$ de altura a más. La copa es de forma columnar a cónica con 6 $\mathrm{m}$ de diámetro. Las hojas son compuestas y de forma característica. Las flores están agrupadas en forma longitudinal al extremo de las ramas. Loa frutos son cápsulas pequeñas y aplanadas. El tronco es recto con una corteza externa fisurada. Las raíces son de crecimiento vertical profundo (Bulnes et al. 2017).

\section{Selección de la muestra}

Para la selección de los individuos a cortar, se realizó un inventario de las especies en el área de estudio, clasificándolos según su categoría diamétrica, con una amplitud de $5 \mathrm{~cm}$. Esta información se utilizó, con la finalidad de obtener una muestra que incluya individuos en las diferentes clases diamétricas (Segura y Andrade 2008).
En este estudio se realizaron ensayos destructivos (Acosta et al. 2002; Ávila et al. 2001; Rugnitz et al. 2009) para 44 individuos arbóreos (Cuadro 1). Todos los árboles fueron seleccionados al azar dentro de cada categoría diamétrica.

Cabe señalar la dificultad de extraer individuos presentes en parques, calles o avenidas para realizar ensayos destructivos, razón por la cual se trabajó con individuos pertenecientes al "Bosque simulado y Plantación forestal". Asimismo, se tuvo la dificultad de extraer un número mayor de individuos, de dicha plantación, ya que son árboles comerciales y solo se podía extraer un número no mayor a 4 individuos por clase diamétrica.

\section{Trabajo de campo}

En cada árbol seleccionado para el ensayo destructivo, se midió el diámetro a la altura del pecho del árbol (DAP) en pie. Para árboles pequeños (diámetro menor a $5 \mathrm{~cm}$ ) se midió el diámetro a $30 \mathrm{~cm}$ del suelo, según lo recomendado por Segura y Andrade (2008). Luego se procedió a cortar el árbol, registrar la altura comercial e identificar el eje principal del tronco, siguiendo las recomendaciones de Picard et al. (2012), principalmente para árboles con troncos muy sinuosos o ramosos (como el caso del Tecoma stans var. stans y el Schinus molle).

Posteriormente el árbol fue dividido en secciones (tronco, hojas y ramas) para registrar el peso húmedo de cada componente. El tronco a su vez fue subdividido en secciones de aproximadamente $1 \mathrm{~m}$ cada una y se registró el peso

\begin{tabular}{|c|c|c|c|c|c|c|c|}
\hline \multirow{2}{*}{$\begin{array}{c}\text { Clase } \\
\text { diamétrica }\end{array}$} & $\begin{array}{c}\text { Rango } \\
\text { diamétrico } \\
(\mathbf{c m})\end{array}$ & \multicolumn{2}{|c|}{ Grevillea robusta } & \multicolumn{2}{|c|}{ Schinus molle } & \multicolumn{2}{c|}{ Tecoma stans } \\
\cline { 3 - 8 } & & Total & Muestra & Total & Muestra & Total & Muestra \\
\hline 1 & $0.1-5$ & 8 & 3 & 10 & 3 & 6 & 3 \\
\hline 2 & $5.1-10$ & 21 & 3 & 29 & 4 & 26 & 4 \\
\hline 3 & $10.1-15$ & 25 & 4 & 55 & 3 & 26 & 4 \\
\hline 4 & $15.1-20$ & 34 & 3 & 31 & 3 & 31 & 4 \\
\hline 5 & $20.1-25$ & 26 & 2 & 36 & 1 & 14 & - \\
\hline
\end{tabular}

Cuadro 1. Cantidad de árboles extraído por especie y rango diamétrico. 

en especies arbóreas de Lima, Perú

húmedo total, asimismo fueron extraídas rodajas de $5 \mathrm{~cm}$ de cada sección para la determinación del contenido de humedad. Las ramas fueron separadas y divididas según diámetro: $<$ a $2 \mathrm{~cm}$, entre 2 y 7 y $>$ a $7 \mathrm{~cm}$, y posteriormente pesadas, extrayéndose además, una rodaja de 5 $\mathrm{cm}$ para el caso de ramas grandes y de $10 \mathrm{~cm}$ para ramas pequeñas para la determinación del contenido de humedad. En el caso de las hojas, estas fueron separadas de las ramas y pesadas en una balanza digital y se extrajo una muestra de $25 \mathrm{~g}$ para la determinación del contenido de humedad.

\section{Determinación del contenido de humedad}

Se empleó el método gravimétrico para la determinación de la humedad según la norma ASTM D4442 (ASTM 2008). Todas las muestras (de tronco, ramas y follaje) fueron secadas en el horno a una temperatura de $103+/-2{ }^{\circ} \mathrm{C}$ hasta alcanzar peso constante. El contenido de humedad $(\mathrm{CH})$ se calculó empleando la siguiente ecuación:

$$
\text { CH }(\%)=\left[\frac{\text { Peso húmedo }- \text { Peso seco }}{\text { Peso seco }}\right] \times 100
$$

\section{Procedimiento para el cálculo de la biomasa aérea}

La biomasa total aérea $(\mathrm{kg})$ se determinó a través de la siguiente ecuación, siguiendo la metodología propuesta por Rugnitz et al. (2009):

$$
\text { Biomasa Total }=B F+B R+B H
$$

Donde:

$$
\begin{aligned}
& \mathrm{BF}=\text { Biomasa Fusta } \\
& \mathrm{BR}=\text { Biomasa Ramas } \\
& \mathrm{BH}=\text { Biomasa Hojas }
\end{aligned}
$$

La biomasa de cada componente (fuste, ramas, hojas) se determinó a través de la siguiente ecuación (Rugnitz et al. 2009):

$$
\text { Biomasa }=\frac{\text { Peso húmedo componente }(\mathrm{kg})}{\left(1+\frac{C H \%}{100}\right)}
$$

\section{Selección de modelos de biomasa aérea}

A partir del DAP $(1.30 \mathrm{~m})$, la altura comercial (hc) y la altura total (ht), se ensayaron modelos alométricos, para la estimación de la biomasa, entre los cuales se utilizaron modelos con una variable (DAP) y dos variables (DAP hc y DAP - ht). Luego se graficó la variable biomasa (dependiente) contra todas las variables independientes (DAP, hc y ht) para observar la tendencia de los datos.

Los diferentes modelos alométricos y los supuestos estadísticos del modelo de regresión fueron estimados con el software E-views 7 . Asimismo, se evaluó el cumplimiento de los supuestos estadísticos del modelo de regresión lineal: homocedasticidad, no multicolinealidad y autocorrelación. Para la detección de heterocedasticidad se utilizó el test de Breusch-Pagan-Godfrey y de White con términos cruzados y sin términos cruzados. En el caso de la detección de autocorrelación se utilizó el Test de Breusch Godfrey, y para la detección de multicolinealidad se usó el Factor de Inflación de la Varianza (FIV).

Para la selección del modelo de mejor ajuste se consideró el Coeficiente de Determinación $\left(\mathrm{R}^{2}\right)$ con valores mayores a 0.8 y para la selección del mejor modelo se trabajó en base a lo recomendado por Segura y Andrade (2008): simplicidad en la obtención de las variables, significancia de los parámetros, el Error Estándar de la Regresión o raíz del cuadrado medio del error (RCME) y el Índice de Furnival (IF). En todas las pruebas de hipótesis se consideró un nivel de significancia de 0.05 . Las fórmulas empleadas para el RCME y el Índice de Furnival (Segura y Andrade 2012) son indicadas a continuación:

- Error Estándar de la Regresión

$$
R C M E=\sqrt{S C R /(n-p)}
$$

Donde:

SCR = Suma del cuadrado de los residuos

$\mathrm{n}=$ Numero de observaciones 
$\mathrm{p}=$ Número de parámetros del modelo lineal (incluyendo el término $\beta$ o)

- Índice de Furnival

$$
I F=R C M E \times \operatorname{antilog}\left[\frac{\sum \log \left(\left(B^{\prime}\right)^{-1}\right)}{n}\right]
$$

Donde:

IF = Índice de Furnival

RCME = Raíz cuadrada del cuadrado medio del error

$\left(B^{\prime}\right)^{-1}=$ Recíproco de la primera derivada de la variable dependiente (transformada)

\section{Resultados}

\section{Estadísticas descriptivas para las variables utilizadas}

Los valores mínimos, máximos, media aritmética y desviación estándar de las variables utilizadas (diámetro a la altura del pecho, altura total y altura comercial), de los valores para biomasa sobre el suelo total y por componente para cada especie, son presentadas en el Cuadro 2. La especie Schinus molle, reportó los valores mínimos y máximos para la variable DAP, variando entre $1.3 \mathrm{~cm}$ y un máximo de $22.9 \mathrm{~cm}$. En el caso de la altura total es la especie Grevillea robusta, la que presenta los valores máximos $(21.4 \mathrm{~m})$ y el valor mínimo lo representa Schinus molle con $2.7 \mathrm{~m}$.

\section{Estimación de la biomasa aérea total}

Con los datos obtenidos de peso húmedo total de cada componente del árbol y el contenido de humedad de las muestras, se determinó la cantidad de biomasa aérea total y por componente para cada especie (Cuadro 3). La biomasa aérea promedio varía entre $32.94 \mathrm{~kg} /$ árbol (S. molle) y $68.49 \mathrm{~kg} /$ árbol (G. robusta). Asimismo se determinó la biomasa por componente encontrándose que es el fuste el que contiene la mayor cantidad de biomasa (56.87 $\%)$ y el que menor cantidad de biomasa contiene serían las hojas (3.94\%).

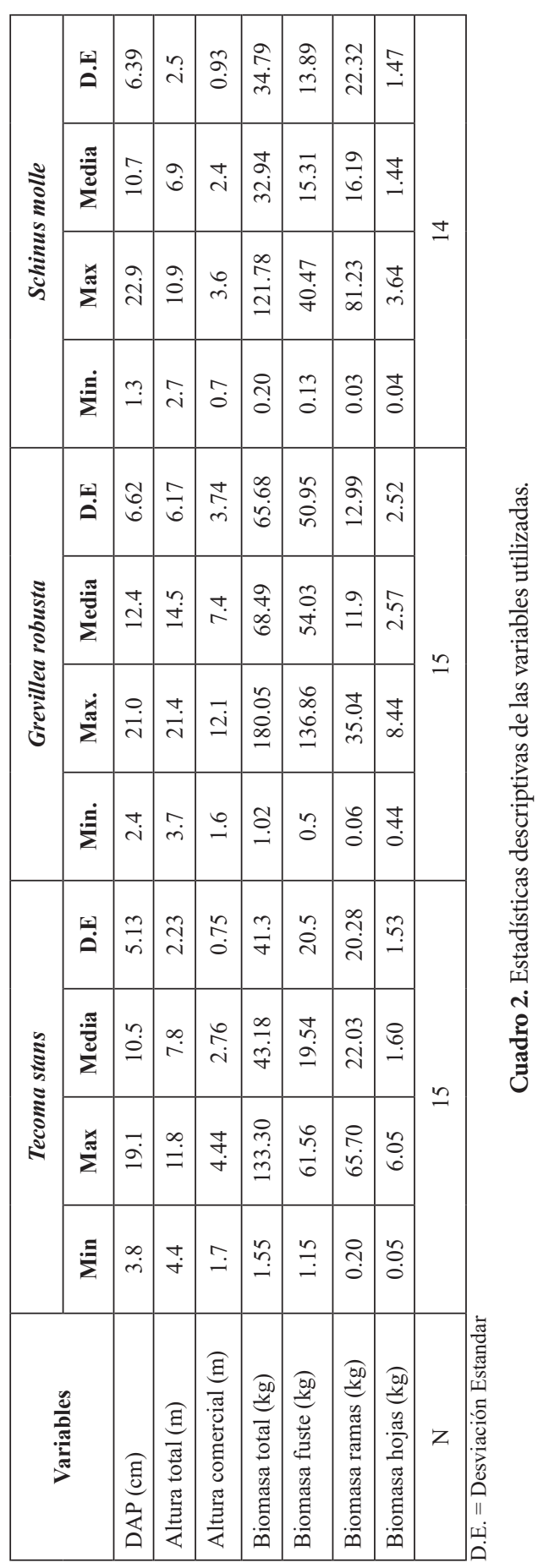



en especies arbóreas de Lima, Perú

\begin{tabular}{|l|c|c|c|c|c|c|c|}
\hline \multirow{2}{*}{\multicolumn{1}{|c|}{ Especie }} & \multicolumn{2}{|c|}{ Fuste } & \multicolumn{2}{c|}{ Ramas } & \multicolumn{2}{c|}{ Hojas } & $\begin{array}{c}\text { Biomasa Total } \\
\text { promedio (kg) }\end{array}$ \\
\cline { 2 - 8 } & Media & $\mathbf{( \% )}$ & Media & $\mathbf{( \% )}$ & Media & $\mathbf{( \% )}$ & 43.18 \\
\hline Tecoma stans & 19.54 & 45.26 & 22.03 & 51.03 & 1.60 & 3.71 & 68.49 \\
\hline Grevillea robusta & 54.03 & 78.88 & 11.9 & 17.37 & 2.57 & 3.75 & 32.94 \\
\hline Schinus molle & 15.31 & 46.48 & 16.19 & 49.15 & 1.44 & 4.37 & - \\
\hline Promedio & 29.63 & 56.87 & 16.71 & 39.18 & 1.87 & 3.94 & - \\
\hline
\end{tabular}

Cuadro 3. Biomasa aérea total $(\mathrm{kg})$ y por componente según especie.

\begin{tabular}{|l|c|c|c|c|c|c|}
\hline Origen de la variación & S. C & G. L & C.M & F & Probabilidad & $\begin{array}{c}\text { Valor } \\
\text { F }\end{array}$ \\
\hline Entre grupos & 9811.93 & 2 & 4905.97 & 2.019 & 0.146 & 3.23 \\
\hline Dentro de los grupos & 99594.35 & 41 & 2428.13 & - & - & - \\
\hline Total & 109406.28 & 43 & - & - & - & - \\
\hline
\end{tabular}

S.C. = Suma de cuadrados; G.L. = Grados de Libertad; C.M. = Cuadrado medio; F = Prueba de Fisher

Cuadro 4. Análisis de varianza para la biomasa sobre el suelo total entre especies.

\begin{tabular}{|l|c|c|c|c|c|c|}
\hline Origen de la variación & S. C & G. L & C.M & F & Probabilidad & $\begin{array}{c}\text { Valor } \\
\text { F }\end{array}$ \\
\hline Entre grupos & 13320.68 & 2 & 6660.34 & 6.098 & 0.0048 & 3.226 \\
\hline Dentro de los grupos & 44779.89 & 41 & 1092.19 & - & - & - \\
\hline Total & 58100.57 & 43 & - & - & - & - \\
\hline
\end{tabular}

S.C. $=$ Suma de cuadrados; G.L. = Grados de Libertad; C.M. = Cuadrado medio; F = Prueba de Fisher

Cuadro 5. Análisis de varianza de la biomasa del fuste entre especies.

\begin{tabular}{|c|c|c|}
\hline \multirow{2}{*}{ Especies } & \multirow{2}{*}{ Valor DSH } & $\begin{array}{c}\text { Diferencias entre } \\
\text { promedios de } \\
\text { biomasa (kg) }\end{array}$ \\
\cline { 3 - 3 } & & Fuste \\
\hline $\mathrm{G}-\mathrm{T}$ & 29.01 & 34.49 \\
\hline $\mathrm{G}-\mathrm{S}$ & 29.53 & 38.71 \\
\hline $\mathrm{T}-\mathrm{S}$ & 29.53 & 4.23 \\
\hline
\end{tabular}

Cuadro 6. Valores encontrados en el Test de Tukey-Kramer de las diferencias entre promedios de biomasa del fuste entre especies.
Para determinar si existe diferencias significativas entre los resultados obtenidos, se realizó el análisis de varianza, encontrándose que no existe diferencias significativas entre los promedios de biomasa aérea total entre especies $(p=0.146)$ (Cuadro 4).

\section{Biomasa sobre el suelo por componentes en- tre especies}

Se comparó la biomasa de las tres especies en estudio según componente del árbol. Del análisis de varianza (Cuadro 5) se encontró que el único componente que presenta diferencias significativas entre las tres especies es el fuste 


\begin{tabular}{|c|c|c|c|c|}
\hline \multirow{2}{*}{ Especie } & \multirow{2}{*}{$\begin{array}{l}\text { Valor } \\
\text { DSH }\end{array}$} & \multicolumn{3}{|c|}{ Diferencias entre promedios de biomasa(kg) } \\
\hline & & Fuste - Ramas & Fuste - Hojas & Ramas - Hojas \\
\hline Tecoma stans & 14.64 & 2.49 & 17.94 & 20.43 \\
\hline Schinus molle & 13.71 & 0.88 & 13.88 & 14.75 \\
\hline Grevillea robusta & 26.68 & 42.13 & 51.46 & 9.33 \\
\hline
\end{tabular}

Cuadro 7. Valores encontrados en el Test de Tukey-Kramer para diferencias entre promedios de biomasa por componente dentro de la especie.

$(p=0.0048)$. No se encontraron diferencias significativas para los componentes ramas $(p=$ $0.337)$ y hojas $(p=0.2133)$ cuando se comparó entre especies.

Asimismo, se realizó el Test de Tukey-Kramer, para identificar cuál especie difiere significativamente de la otra en cuanto al contenido de biomasa en el fuste, siendo la G. robusta, la especie que difiere significativamente de las demás (Cuadro 6).

\section{Biomasa sobre el suelo por componente den- tro de la misma especie}

Por medio del análisis de varianza, se encontró que existen diferencias estadísticamente significativas, entre la biomasa por componentes dentro de cada especie: T. stans var. stans ( $p$ $=0.0029), S$. molle $(p=0.02), G$. robusta $(p=$ 0.00006 ).

Asimismo se utilizó el test de Tukey-Kramer (Diferencias Honestamente significativas - DSH) para determinar que componente difiere significativamente del resto (Cuadro 7), encontrándose que para el caso de T. stans var. stans y $S$. molle, el componente que difiere son las hojas. En el caso de G. robusta, es el fuste el componente que difiere significativamente en relación a la cantidad de biomasa.

\section{Selección de modelos de biomasa aérea}

Los modelos que presentaron mejor ajuste fueron los que contenían solo al DAP. Se analizaron solo aquellos modelos de mejor ajuste y cuyo coeficiente de determinación fuera superior a 0.8. Tanto los parámetros (Prueba T-stu- dent) como el modelo seleccionado fueron significativos (Prueba Fisher) para un nivel de $5 \%$.

\section{Selección de la mejor ecuación de biomasa aérea para $T$. stans}

Fueron evaluados 14 modelos, verificándose la significancia del modelo (prueba F) y de los parámetros (Prueba T-student) (Cuadro 8). Las ecuaciones seleccionadas para $T$. stans superan los valores de $0.86 \mathrm{R}^{2}$. Tanto los parámetros como la ecuación seleccionada fueron significativos (Cuadro 9). La ecuación para la especie es un modelo linealizado de la forma $\operatorname{Ln}(B T)=-2.9409+2.6925 \times \operatorname{Ln}($ dap $)$ que presenta un coeficiente de determinación de $\mathrm{R}^{2}=$ 0.93, lo cual indica que la ecuación tiene buen ajuste, ya que el valor es mayor a 1, en concreto 93\% de la variabilidad de la biomasa es explicada por el modelo de regresión. En el caso del Índice de Furnival este presenta el valor más bajo $(\mathrm{IF}=9.00)$.

\section{Selección de la mejor ecuación de biomas aérea para para G. robusta}

El DAP fue la variable independiente más correlacionada con la biomasa aérea total ( $\mathrm{r}$ $=0.94$ ). Las ecuaciones seleccionadas para $G$. robusta superan los valores de $0.97 \mathrm{R}^{2}$ (Cuadro 10). Tanto los parámetros como el modelo fueron significativos (Cuadro 11). La ecuación seleccionada, para la especie es un modelo linealizado de la forma $\operatorname{Ln}(B T)=-2.0082+$ $2.3293 \times \operatorname{Ln}($ dap $)$ que presenta un $\mathrm{R}^{2}=0.99$, lo cual indica que la ecuación tiene buen ajuste, ya que el valor es mayor a 1 , en concreto $99 \%$ 
de la variabilidad de la biomasa es explicada por el modelo de regresión. Los resultados para el Índice de Furnival son los más bajos para esta ecuación $(\mathrm{IF}=3.53)$.

\section{Selección de modelos de biomasa aérea para S. molle}

El DAP fue la variable independiente más correlacionada con la biomasa aérea total ( $\mathrm{r}$ $=0.94)$. Las ecuaciones seleccionadas para $S$. molle superan los valores de $0.97 \mathrm{R}^{2}$ (Cuadro
12), y tanto los parámetros como la ecuación son significativos (Cuadro 13). El modelo seleccionado, para la especie es un modelo linealizado de la forma $\operatorname{Ln}(B T)=-3.3201+1.4834$ $\times \operatorname{Ln}\left(\right.$ dap) que presenta un $\mathrm{R}^{2}=0.99$ lo cual indica que la ecuación tiene buen ajuste, ya que el valor es mayor a 1 , en concreto $99 \%$ de la variabilidad de la biomasa es explicada por el modelo de regresión. En el caso del Índice de Furnival este presenta el valor más bajo (IF = 2.22).

\begin{tabular}{|c|c|c|c|c|c|}
\hline \multirow{2}{*}{ Modelo } & \multicolumn{2}{|c|}{$\mathbf{R}^{2}$} & \multirow{2}{*}{$\begin{array}{c}\mathbf{T} \\
(p-v a l u e)\end{array}$} & \multirow{2}{*}{$\begin{array}{c}\mathrm{F} \\
(p-v a l u e)\end{array}$} & \multirow{2}{*}{ Condición } \\
\hline & Valor & Ajuste & & & \\
\hline $\mathrm{B}=\mathrm{b} 0+\mathrm{b} 1 \mathrm{dap}$ & 0.89 & $\mathrm{Si}$ & $* *$ & $* *$ & Aceptado \\
\hline $\mathrm{B}=\mathrm{b} 0+\mathrm{b} 1 \mathrm{dap}^{2}$ & 0.9275 & $\mathrm{Si}$ & $* *$ & $* *$ & Aceptado \\
\hline $\mathrm{B}=\mathrm{b} 0+\mathrm{b} 1 \mathrm{dap}+\mathrm{b} 2 \mathrm{dap}^{2}$ & 0.9277 & $\mathrm{Si}$ & No & $* *$ & Rechazado \\
\hline $\mathrm{B}=\mathrm{b} 0+\mathrm{b} 1 \mathrm{dap}^{2}+\mathrm{b} 2 \mathrm{ht}$ & 0.9278 & $\mathrm{Si}$ & No & $* *$ & Rechazado \\
\hline $\mathrm{B}=\mathrm{b} 0+\mathrm{b} 1\left(\mathrm{dap}^{2} \times \mathrm{ht}\right)$ & 0.8639 & $\mathrm{Si}$ & $* *$ & $* *$ & Aceptado \\
\hline $\mathrm{B}=\mathrm{b} 0+\mathrm{b} 1 \ln (\mathrm{dap})$ & 0.7842 & No & $* *$ & $* *$ & Rechazado \\
\hline $\mathrm{B}=\mathrm{b} 0+\mathrm{b} 1 \ln (\mathrm{dap})+\mathrm{b} 2 \ln (\mathrm{ht})$ & 0.8022 & $\mathrm{Si}$ & No & $* *$ & Rechazado \\
\hline $\ln B=b 0+b 1 \ln (d a p)$ & 0.9259 & $\mathrm{Si}$ & $* *$ & $* *$ & Aceptado \\
\hline $\ln B=b 0+b 1 \ln ($ dap $\times h t)$ & 0.9052 & $\mathrm{Si}$ & $* *$ & $* *$ & Aceptado \\
\hline $\ln B=b 0+b 1 \ln \left(\right.$ dap $\left.^{2} \times h t\right)$ & 0.9248 & $\mathrm{Si}$ & $* *$ & $* *$ & Aceptado \\
\hline $\begin{array}{c}\mathrm{B}=\mathrm{b} 0+ \\
+b 1 \text { dap }+ \text { b2 }(\text { dap } \times \text { ht })+b 3 \text { dap }^{2} \\
+b 4 \text { ht }+ \text { b5 }\left(\text { dap }^{2} \times \text { ht }\right.\end{array}$ & 0.936 & $\mathrm{Si}$ & No & $* *$ & Rechazado \\
\hline $\mathrm{B}=\mathrm{b} 0+\mathrm{b} 1 \mathrm{dap}+\mathrm{b} 2 \mathrm{ht}$ & 0.8393 & $\mathrm{Si}$ & No & $* *$ & Rechazado \\
\hline $\mathrm{B}=\mathrm{b} 0+\mathrm{b} 1 \mathrm{dap}+\mathrm{b} 2 \mathrm{dap}^{2}+\mathrm{b} 3 \mathrm{ht}$ & 0.9277 & $\mathrm{Si}$ & No & $* *$ & Rechazado \\
\hline $\ln B=b 0+b 1 \ln ($ dap $)+b 2 \ln (h t)$ & 0.9295 & $\mathrm{Si}$ & No & $* *$ & Rechazado \\
\hline
\end{tabular}

Cuadro 8. Modelos utilizados y condición de aceptación o rechazo para la estimación de la biomasa aérea de Tecoma stans. Fuente: Segura y Andrade (2008), Ruiz (2007) y Picard et al. (2012). 


\begin{tabular}{|c|c|c|c|c|c|}
\hline \multirow{2}{*}{ Modelo } & \multicolumn{2}{|c|}{ Parámetros } & \multirow{2}{*}{$\mathbf{R}^{2}$} & \multirow{2}{*}{ RCME } & \multirow{2}{*}{ IF } \\
\hline & $\beta 0$ & $\beta 1$ & & & \\
\hline$B=b 0+b 1$ dap & -36.6205 & 7.6011 & 0.89 & 14.21 & 14.21 \\
\hline$B=b 0+b 1 d a p^{2}$ & -2.5918 & 0.3397 & 0.93 & 11.54 & 11.54 \\
\hline$B=b 0+b 1\left(d_{a p}^{2} \times h t\right)$ & 3.5949 & 0.0326 & 0.86 & 15.81 & 15.81 \\
\hline $\ln (B)=b 0+b 1 \ln (d a p)$ & -2.9409 & 2.6935 & 0.93 & 0.42 & 9.00 \\
\hline $\ln (B)=b 0+b 1 \ln ($ dap $\times h t)$ & -4.5823 & 1.8002 & 0.91 & 0.48 & 10.18 \\
\hline $\ln (B)=b 0+b 1 \ln \left(d a p^{2} \times h t\right)$ & -4.0109 & 1.0923 & 0.92 & 0.43 & 9.07 \\
\hline
\end{tabular}

$\mathrm{R}^{2}=$ Coeficiente de determinación; RCME = Raíz del cuadrado medio del error; IF= Índice de Furnival.

Cuadro 9. Modelos alométricos seleccionados y estadígrafos obtenidos para la biomasa aérea de T. stans

\begin{tabular}{|c|c|c|c|c|c|}
\hline \multirow{2}{*}{ Modelo } & \multicolumn{2}{|c|}{$\mathbf{R}^{2}$} & \multirow{2}{*}{$\begin{array}{c}\mathrm{T} \\
(p-v a l u e)\end{array}$} & \multirow{2}{*}{$\begin{array}{c}\mathrm{F} \\
(p \text {-value })\end{array}$} & \multirow{2}{*}{ Condición } \\
\hline & Valor & Ajuste & & & \\
\hline $\mathrm{B}=\mathrm{b} 0+\mathrm{b} 1 \mathrm{dap}$ & 0.89129 & $\mathrm{Si}$ & $* *$ & $* *$ & Aceptado \\
\hline $\mathrm{B}=\mathrm{b} 0+\mathrm{b} 1 \mathrm{dap}^{2}$ & 0.965 & $\mathrm{Si}$ & $* *$ & ** & Aceptado \\
\hline $\mathrm{B}=\mathrm{b} 0+\mathrm{b} 1$ dap $+\mathrm{b} 2 \mathrm{dap}^{2}$ & 0.9771 & $\mathrm{Si}$ & No & $* *$ & Rechazado \\
\hline $\mathrm{B}=\mathrm{b} 0+\mathrm{b} 1 \mathrm{dap}^{2}+\mathrm{b} 2 \mathrm{ht}$ & 0.96919 & $\mathrm{Si}$ & No & $* *$ & Rechazado \\
\hline $\mathrm{B}=\mathrm{b} 0+\mathrm{b} 1\left(\mathrm{dap}^{2} \times \mathrm{ht}\right)$ & 0.9808 & $\mathrm{Si}$ & $* *$ & $* *$ & Aceptado \\
\hline $\mathrm{B}=\mathrm{b} 0+\mathrm{b} 1 \ln (\mathrm{dap})$ & 0.72289 & No & $* *$ & $* *$ & Rechazado \\
\hline $\mathrm{B}=\mathrm{b} 0+\mathrm{b} 1(\mathrm{dap} \times \mathrm{ht})$ & 0.9309 & $\mathrm{Si}$ & $* *$ & $* *$ & Aceptado \\
\hline $\ln \mathrm{B}=\mathrm{b} 0+\mathrm{b} 1 \ln (\mathrm{dap})$ & 0.9953 & $\mathrm{Si}$ & $* *$ & $* *$ & Aceptado \\
\hline $\ln B=b 0+b 1 \ln (d a p \times h t)$ & 0.9778 & $\mathrm{Si}$ & $* *$ & $* *$ & Aceptado \\
\hline $\ln \mathrm{B}=\mathrm{b} 0+\mathrm{b} 1 \ln \left(\mathrm{dap}^{2} \times \mathrm{ht}\right)$ & 0.9876 & $\mathrm{Si}$ & $* *$ & $* *$ & Aceptado \\
\hline $\mathrm{B}=\mathrm{b} 0+\mathrm{b} 1 \mathrm{dap}^{2}+\mathrm{b} 2 \mathrm{hc}$ & 0.967 & $\mathrm{Si}$ & No & $* *$ & Rechazado \\
\hline $\mathrm{B}=\mathrm{b} 0+\mathrm{b} 1 \ln (\mathrm{dap})+\mathrm{b} 2 \ln (\mathrm{hc})$ & 0.73 & No & No & $* *$ & Rechazado \\
\hline $\ln B=b 0+b 1 \ln \left(d a p^{2} \times h t\right)+b 2 \ln (h c)$ & 0.9917 & $\mathrm{Si}$ & $* *$ & ** & Aceptado \\
\hline $\ln B=b 0+b 1 \ln (d a p)+b 2 \ln (h t)$ & 0.995 & $\mathrm{Si}$ & No & $* *$ & Rechazado \\
\hline
\end{tabular}

$\mathrm{F}=$ Prueba de Fisher; $\mathrm{t}=$ Prueba T-student; $* *$ nivel de significancia al 5\%.

Cuadro 10. Modelos utilizados y condición de aceptación o rechazo para la estimación de la biomasa aérea de Grevillea robusta. Fuente: Segura y Andrade (2008), Ruiz (2007) y Picard et al. (2012). 

en especies arbóreas de Lima, Perú

\begin{tabular}{|c|c|c|c|c|c|c|}
\hline \multirow{2}{*}{ Modelo } & \multicolumn{3}{|c|}{ Parámetros } & \multirow{2}{*}{$\mathbf{R}^{2}$} & \multirow{2}{*}{ RCME } & \multirow{2}{*}{ IF } \\
\hline & $\boldsymbol{\beta}_{0}$ & $\beta_{1}$ & $\boldsymbol{\beta}_{2}$ & & & \\
\hline$B T=b 0+b 1$ dap & -47.67 & 9.3638 & - & 0.89 & 22.47 & 22.47 \\
\hline$B T=b 0+b 1 d a p^{2}$ & -9.3239 & 0.3994 & - & 0.97 & 12.75 & 12,75 \\
\hline$B T=b 0+b 1\left(d a p^{2} \times h t\right)$ & -0.4542 & 0.0189 & - & 0.98 & 9.44 & 9.44 \\
\hline$B T=b 0+b 1($ dap $\times h t)$ & -17.018 & 0.3966 & - & 0.93 & 17.92 & 17.92 \\
\hline $\ln (B T)=b 0+b 1 \ln ($ dap $)$ & -2.0082 & 2.3293 & - & 0.99 & 0.12 & 3.53 \\
\hline $\ln (B T)=b 0+b 1 \ln (d a p \times h t)$ & -2.819 & 1.2792 & - & 0.98 & 0.25 & 7.75 \\
\hline $\ln (B T)=b 0+b 1 \ln \left(d a p^{2} \times h t\right)$ & -2.5533 & 0.8287 & - & 0.99 & 0.19 & 5.79 \\
\hline $\ln (B T)=b 0+b 1 \ln \left(d a p^{2} \times h t\right)+b 2 \ln$ & -2.6915 & 0.9351 & -0.3439 & 0.99 & 0.16 & 4.91 \\
\hline
\end{tabular}

$\mathrm{R}^{2}=$ Coeficiente de determinación; RCME = Raíz del cuadrado medio del error; IF= Índice de Furnival.

Cuadro 11. Modelos alométricos y estadísticos obtenidos para la biomasa aérea de Grevillea robusta.

\section{Discusión}

Los resultados obtenidos para biomasa aérea de tres especies forestales y los modelos presentados pueden ser utilizados para estimar directamente la cantidad de carbono almacenado, independientemente de las características del sitio, como lo sugiere Fortier et al. (2017), quien indica que la determinación de modelos alométricos depende del objetivo de la investigación, pudiendo ser utilizado los modelos generales cuando el objetivo es estimaciones de biomasa con fines de captura de carbono.

De acuerdo al Análisis de varianza (Cuadro 7) no existen diferencias significativas entre los promedios de biomasa total entre especies. Estos resultados se deben a la variabilidad en los datos obtenidos a razón de las diferencias en la arquitectura de las especies. En cuanto a las cantidades de biomasa por componente entre especies, el tronco de la especie G. robusta es el componente que difiere entre las especies. Fritzons et al. (2014), señalan que G. robusta es una especie heliófita de lento crecimiento y que tiende a acumular mayor cantidad de carbono en el fuste para poder acceder a una mayor cantidad de luz en el dosel, esto es porque posee un fuste de crecimiento monopodial.
La distribución de biomasa entre componentes para la especie G. robusta obtenidos en la presente investigación concuerdan con los de otros autores como Fonseca et al. (2011), Gayoso y Guerra (2005), Monroy y Návar (2004), Soriano-Luna (2015), Pérez y Kanninen (2002, citado por González 2008), quienes han señalado que existen diferencias entre la biomasa de los componentes del árbol, y que la mayor proporción de biomasa y carbono en latifoliadas se concentra en el fuste. Estas diferencias pueden deberse a que la proporción de la biomasa en los componentes estructurales de un árbol se modifican en función de la edad, tamaño y patrón de crecimiento (Gayoso 2002). Asimismo, esto puede atribuirse al cierre de copas, en ese caso los árboles grandes tienen un área foliar relativamente menor que los árboles pequeños debido a las limitaciones impuestas por la luz (Lim et al. 2013).

En relación a la cantidad de biomasa por componentes para T. stans var. stans y S. molle, no se encontraron diferencias significativas entre la biomasa del fuste y las ramas, lo cual difiere de lo reportado por Álvarez (2008), Gonzales (2008), Segura (2005), Avendaño (2008), Fonseca et al. (2011), Gayoso y Guerra (2005), Monroy y Navar (2004), Soriano Luna 


\begin{tabular}{|c|c|c|c|c|c|}
\hline \multirow{2}{*}{ ModelO } & \multicolumn{2}{|c|}{$\mathbf{R}^{2}$} & \multirow{2}{*}{$\begin{array}{c}\mathrm{T} \\
(p-v a l u e)\end{array}$} & \multirow{2}{*}{$\begin{array}{c}\text { F } \\
(p-v a l u e)\end{array}$} & \multirow{2}{*}{ Condición } \\
\hline & Valor & Ajuste & & & \\
\hline $\mathrm{B}=\mathrm{b} 0+\mathrm{b} 1 \mathrm{dap}$ & 0.8791 & $\mathrm{Si}$ & $* *$ & $* *$ & Aceptado \\
\hline $\mathrm{B}=\mathrm{b} 0+\mathrm{b} 1 \mathrm{dap}^{2}$ & 0.9668 & $\mathrm{Si}$ & $* *$ & $* *$ & Aceptado \\
\hline $\mathrm{B}=\mathrm{b} 0+\mathrm{b} 1$ dap $+\mathrm{b} 2 \mathrm{dap}^{2}$ & 0.969 & $\mathrm{Si}$ & No & $* *$ & Rechazado \\
\hline $\mathrm{B}=\mathrm{b} 0+\mathrm{b} 1 \mathrm{dap}^{2}+\mathrm{b} 2 \mathrm{ht}$ & 0.9692 & $\mathrm{Si}$ & No & $* *$ & Rechazado \\
\hline $\mathrm{B}=\mathrm{b} 0+\mathrm{b} 1 \mathrm{dap}^{2} \times \mathrm{ht}$ & 0.96608 & $\mathrm{Si}$ & $* *$ & $* *$ & Aceptado \\
\hline $\mathrm{B}=\mathrm{b} 0+\mathrm{b} 1 \ln (\mathrm{dap})$ & 0.602 & No & No & $* *$ & Rechazado \\
\hline $\ln B=b 0+b 1 \ln \left(\right.$ dap $\left.^{2} \times \mathrm{hc}\right)$ & 0.948 & $\mathrm{Si}$ & $* *$ & $* *$ & Aceptado \\
\hline $\ln \mathrm{B}=\mathrm{b} 0+\mathrm{b} 1 \ln (\mathrm{dap})$ & 0.9863 & $\mathrm{Si}$ & $* *$ & $* *$ & Aceptado \\
\hline $\ln B=b 0+b 1 \ln (d a p \times h t)$ & 0.9926 & $\mathrm{Si}$ & $* *$ & $* *$ & Aceptado \\
\hline $\ln \mathrm{B}=\mathrm{b} 0+\mathrm{b} 1 \ln \left(\mathrm{dap}^{2} \times \mathrm{ht}\right)$ & 0.9924 & $\mathrm{Si}$ & $* *$ & $* *$ & Aceptado \\
\hline $\mathrm{B}=\mathrm{b} 0+\mathrm{b} 1 \mathrm{dap}^{2}+\mathrm{b} 2 \mathrm{hc}$ & 0.969 & $\mathrm{Si}$ & No & $* *$ & Rechazado \\
\hline $\mathrm{B}=\mathrm{b} 0+\mathrm{b} 1 \ln (\mathrm{dap})+\mathrm{b} 2 \ln (\mathrm{hc})$ & 0.63 & No & No & $* *$ & Rechazado \\
\hline $\ln B=b 0+b 1 \ln \left(d_{a p}^{2} \times h c\right)+b 2 \ln (h c)$ & 0.987 & $\mathrm{Si}$ & $* *$ & $* *$ & Aceptado \\
\hline $\ln \mathrm{B}=\mathrm{b} 0+\mathrm{b} 1 \ln (\mathrm{dap})+\mathrm{b} 2 \ln (\mathrm{ht})$ & 0.9929 & $\mathrm{Si}$ & No & $* *$ & Rechazado \\
\hline
\end{tabular}

$\mathrm{F}=$ Prueba de Fisher; $\mathrm{t}$ = Prueba T-student; $* *$ nivel de significancia al 5\%.

Cuadro 12. Modelos utilizados y condición de aceptación o rechazo para la estimación de la biomasa aérea de Schinuns molle. Fuente: Segura y Andrade (2008), Ruiz (2007) y Picard et al. (2012).

(2015), quienes indican una mayor proporción de biomasa y carbono en el fuste, seguido por las ramas y las hojas. Esto se puede explicar debido a la estructura fenotípica de las especies, las cuales presentan un crecimiento simpodial, donde las ramas presentan un desarrollo igual o superior que el eje principal en comparación con aquellas del tipo monopodial (como en G. robusta). Por su parte Xiao (1998) y Brack (2002, citado por Aguaron y McPherson 2012) indican un mayor contenido de carbono en las ramas y hojas en comparación al fuste.
Del mismo modo, las especies que tienden a crecer en espacios abiertos, desarrollan una mayor copa y con mayor cantidad de ramas (Johnson y Gerhold 2001), como es el caso de las especies en estudio, dado que no tienen competencia por luz, como aquellas que se desarrollan en un bosque natural o en plantaciones, de donde además proviene la mayor parte de las investigaciones.

Los resultados obtenidos indican una gran correlación entre la biomasa y el diámetro. Esto se explica, ya que el nivel de desarrollo 

en especies arbóreas de Lima, Perú

\begin{tabular}{|c|c|c|c|c|c|c|}
\hline \multirow{2}{*}{ Modelo } & \multicolumn{3}{|c|}{ Parámetros } & \multirow{2}{*}{$\mathbf{R}_{2}$} & \multirow{2}{*}{ RCME } & \multirow{2}{*}{ IF } \\
\hline & $\boldsymbol{\beta}_{0}$ & $\beta_{1}$ & $\boldsymbol{\beta}_{2}$ & & & \\
\hline$B T=b o+b 1 d a p$ & -20.9 & 5.0372 & - & 0.88 & 12.42 & 12.42 \\
\hline$B T=b o+b 1 d a p^{2}$ & -1.0413 & 0.2233 & - & 0.97 & 6.51 & 6.51 \\
\hline$B T=b o+b 1\left(d a p^{2} \times h t\right)$ & 4.5783 & 0.0208 & - & 0.97 & 6.68 & 6.58 \\
\hline $\ln (B)=b o+b 1 \ln \left(d_{a p}^{2} \times h c\right)$ & -2.1694 & 0.9459 & - & 0.95 & 0.4339 & 5.88 \\
\hline $\ln (B T)=b 0+b 1 \ln (d a p)$ & -2.0465 & 2.1906 & - & 0.99 & 0.22 & 3.02 \\
\hline $\ln (B T)=b 0+b 1 \ln (d a p \times h t)$ & -3.3201 & 1.4834 & - & 0.99 & 0.16 & 2.22 \\
\hline $\ln (B T)=b 0+b 1 \ln \left(d a p^{2} \times h t\right)$ & -2.8186 & 0.8866 & - & 0.99 & 0.17 & 2.25 \\
\hline $\begin{array}{c}\ln (B T)=b 0+b 1 \ln \left(d a p^{2} \times h c\right)+b 2 \ln \\
(h c)\end{array}$ & -2.1025 & 1.0794 & -0.9256 & 0.99 & 0.22 & 2.99 \\
\hline
\end{tabular}

$\mathrm{R}^{2}=$ Coeficiente de determinación; RCME = Raíz del cuadrado medio del error; IF= Índice de Furnival.

Cuadro 13. Modelos alométricos y estadísticos obtenidos para la biomasa aérea de Schinus molle.

de los árboles se ve reflejado tanto en diámetro como en altura, lo que influye directamente en la ganancia de biomasa, esto coincide con lo mencionado por Álvarez (2008) y Arreaga (2002) quienes mencionan que la influencia del incremento del diámetro tiene una relación positiva con respecto al incremento de la biomasa siguiendo una tendencia de crecimiento de tipo ascendente.

El modelo de biomasa seleccionado para T. stans, coincide con lo encontrado por Onyekwelu (2004), en un estudio realizado 120 árboles de Gmelina arborea Roxb. ex Sm. en Nigeria, con edades entre los 5 y los 21 años, logró un $\mathrm{R}^{2}$ de 0.989 para el mismo modelo: $\operatorname{Ln}(\mathrm{Bt})=\mathrm{a}+\mathrm{b} \operatorname{Ln}($ dap). Esto se debería a las similitudes entre ambas especies, como: el rápido crecimiento, el tipo de manejo (plantación forestal) y la edad.

Cabe indicar, que las ecuaciones seleccionadas son modelos linealizados y que si bien la transformación logarítmica corrige la heterogeneidad de la varianza de Y con respecto a $\beta X$ (Brown et al. 1989, citado por Méndez et al. 2012), al incrementar la validez estadística del modelo debido a la homogenización de la varianza (Sprugel 1983; Zianis y Mencuccini 2004, citado por Méndez et al. 2012), cuando las unidades originales son re-transformadas en el modelo, los valores de Y son subestimados (Brown et al. 1989, citado por Méndez et al. 2012).

Las ecuaciones presentadas permiten incrementar el conocimiento sobre biomasa en áreas urbanas en Lima. Sin embargo, se recomienda evaluar modelos que permitan estimar la cantidad de biomasa fijada en respuesta a los distintos manejos silviculturales y ubicación de los árboles (parques, jardines, avenidas etc).

\section{Conclusiones}

La biomasa aérea total promedio para la muestra empleada en el estudio, fue de 43.18 $\mathrm{kg} / a ́ r b o l$ para Tecoma stans var. stans, $68.49 \mathrm{~kg} /$ árbol para Grevillea robusta y 32.94 kg/árbol para Schinus molle, no encontrándose diferencias estadísticamente significativas entre las tres especies.

Existen diferencias significativas entre la biomasa por componentes entre especies, siendo el fuste el único componente que presenta diferencias significativas entre las tres especies. Las ramas y las hojas no presentaron diferencias significativas entre especies.

En el caso de la biomasa por componentes dentro de la misma especie, para Grevillea robusta, se encontró que el fuste es el compo- 
nente que contiene la mayor cantidad de biomasa, en comparación con hojas y ramas, no existiendo diferencias estadísticas entre estos dos componentes. Para Tecoma stans var. stans y Schinus molle, la biomasa del fuste no difiere de la biomasa de ramas, ambos componentes contienen la mayor proporción de biomasa, en comparación con las hojas.

Los modelos alométricos seleccionados para medir la biomasa aérea total fueron $\operatorname{Ln}(B T)=$ $-2.9409+2.6925 \times \operatorname{Ln}(\mathrm{dap})$ para Tecoma stans var. stans, $\operatorname{Ln}(B T)=-2.0082+2.3293 \times \operatorname{Ln}($ dap $)$ para Grevillea robusta y $\operatorname{Ln}(B T)=-3.3201+$ $1.4834 \times \operatorname{Ln}($ dap $\times$ ht $)$ para Schinus molle.

La variable independiente que mejor explicó la biomasa aérea fue el diámetro a la altura del pecho (DAP) para el caso de Tecoma stans var. stans y Grevillea robusta, y la combinación DAP y altura total (ht) para Schinus molle. Estas variables son de fácil medición en campo y muestran una alta correlación con la biomasa.

\section{Agradecimientos}

Los autores expresan sus agradecimientos al Vice-rectorado Académico de la Universidad Nacional Agraria La Molina (UNALM), en especial al Ing. Ignacio Lombardi Indacochea, Vice-rector Académico (2015) y al Vivero de la Facultad de Ciencias Forestales por brindar las facilidades y financiamiento para la realización del presente trabajo.

\section{Bibliografía}

Acosta, M; Vargas, J; Velásquez, A; Etchevers, J. 2002. Estimación de la biomasa aérea mediante el uso de relaciones alométricas en seis especies arbóreas en Oaxaca, México. Agrociencia. $36(6): 725-736$.

Aguaron, E; McPherson, E. 2012. Comparison of Methods for Estimating Carbon Dioxide Storage by Sacramento's Urban Forest. In Lal, R; Augustin, B (eds). Carbon Sequestration in Urban Ecosystems. Ohio, Estados Unidos de Norte América, Springer. p 43-72.

Álvarez, G. 2008. Modelos alométricos para la estimación de biomasa aerea de dos especies nativas en plantaciones forestales del trópico de Cochabamba, Bolivia. Tesis Mg. Sc. Turrialba, Costa Rica, CATIE. 76 p.

Arreaga, W. 2002. Almacenamiento de carbono en bosques con manejo forestal en la Reserva de la Biosfera Maya, Petén, Guatemala. Tesis Mag. Sc. CATIE, Turrialba, Costa Rica. 86 p.

(ASTM) American Society for Testing and Materials. 2008. Annual Book of ASTM standards. Standard test methods for direct moisture content measurement of wood and wood base materials. Norma D 4442-07. 2007. Baltimore, Estados Unidos. 15 Nov.

Ávila, G; Jimenez, F; Beer, J; Gómez, M; Ibrahim, M. 2001. Almacenamiento, fijación de carbono y valoración de servicios ambientales en sistemas agroforestales en Costa Rica. Agroforestería en las Américas 8(30): 32-35.

Bulnes, F; Orrego, M; Terán, A. 2017. Árboles y palmeras del vivero forestal. Características, requerimientos y recomendaciones de 40 especies para la arboricultura urbana. Lima, Perú. 166 p.

Chirinos, A. 2005. Evaluación de diferentes sistemas de fertilización orgánica-mineral en el cultivo de algodón Tanguis var UNA-Centenario. Tesis Ing. Lima, Perú, UNALM. 120 p.

Ferrini, F; Fini, A. 2011. Sustainable management techniques for trees in the urban areas. Journal of Biodiversity and Ecological Sciences 1(1):1-20.

Fonseca, W; Rey, JM; Alice, F. 2011. Carbon accumulation in the biomass and soil of different aged secondary forests in the humid tropics of Costa Rica. Forest Ecology and Management 262 (2011):1400-1408.

Fortier, J; Truax, B; Gagnon, D; Lambert, F. 2017. Allometric Equations for Estimating Compartment Biomass and Stem Volume in Mature Hybrid Poplars: General or Site-Specific?. Forest 8(9):1-23.

Fritzons, E; Povoa de Mattos, P; de Aguiar, A; Muñoz Braz, E; Grabias, J; Ferraz, M. 2014. Crescimento da Grevillea robusta em diferentes sítios edafoclimáticos no Estado do Paraná. Scientia Forestalis 42(103):383-392. 
Gayoso, J. 2002. Medición de la captura de carbono en bosques de Chile y promoción en el mercado mundial. Revista Forestal Iberoamericana 1:1-13.

Gayoso, J; Guerra, J. (2005). Contenido de carbono en la biomasa aérea de bosques nativos en Chile. Bosque 26(2):33-38.

González, M. 2008. Estimación de la biomasa aérea y la captura de carbono en regeneración natural de Pinus maximinoi H. E. Moore, Pinus oocarpa var. ochoterenai Mtz. y Quercus sp. en el norte del Estado de Chiapas, México. Tesis MgSc. Turrialba, Costa Rica, CATIE. 81 p.

Johnson, A; Gerhold, H. 2001. Carbon storage by utility compatible trees. Journal of Arboriculture 27(2):57-68.

Lim, H., K.-H. Lee, K. H. Lee and I. H. Park. 2013. Biomass expansion factors and allometric equations in an age sequence for Japanese cedar (Cryptomeria japonica) in southern Korea. J. For. Res. 18:316-322.

Méndez, J; Turlan, O; Ríos, J; Nájera, J. 2012. Ecuaciones alométricas para estimar la biomasa aérea de Prosopis laevigata (Humb. \& Bonpl. ex Willd.) M.C. Johnst. Revista Mexicana de Ciencias Forestales 3(13):57-72.

Monroy, C; Návar, J. 2004. Ecuaciones de aditividad para estimar componentes de biomasa de Hevea brasiliensis Muell. Arg., en Veracruz, México. Madera y Bosques 10(2): 29-43.

Nowak, D.J. 1994. Atmospheric Carbon Dioxide Reduction by Chicago's Urban Forest. In: Chicago's Urban Forest Ecosystem: Results of the Chicago Urban Forest Climate Project. Gen. Tech. Rep. NE-186. Radnor, PA: U.S. Department of Agriculture, Forest Service, Northeastern Forest Experiment Station. 83-94.

Onyekwelu, J. 2004. Above-ground biomass production and biomass equations for evenaged Gmelina arborea (ROXB) plantations in south-western Nigeria. Biomass and Bioenergy 26: 39-46.

Picard, N; Saint-André, L; Henry, M. 2012. Manual de construcción de ecuaciones alométricas para estimar el volumen y la bio- masa de los árboles: del trabajo de campo a la predicción. Rome, Italia. $223 \mathrm{p}$.

Rügnitz, M; Chacón, M; Porro, R. 2009. Guía para la Determinación de Carbono en Pequeñas Propiedades Rurales. 1. ed. Lima, Perú.: Centro Mundial Agroflorestal (ICRAF) / Consórcio Iniciativa Amazónica (IA). 79 p.

Ruiz, E. 2007. Elaboración de una ecuación de volumen para un bosque de terrazas alta en el Codo del Pozuzo, Puerto Inca, Huánuco. Tesis Ing. Lima, Peru, UNALM. 100p.

Segura, N; Andrade, H. 2008. ¿Cómo construir modelos alométricos de volumen, biomasa y carbono de especies leñosas perennes? Agroforestería en las Américas (46):89-96.

Soriano-Luna, MA; Ángeles-Pérez, G; Martinez-Trinidad; T; Plascencia_Escalante, F; Razo-Zárate; R. 2015. Estimación de biomasa aérea por componente estructural en Zacualtipán, Hidalgo, México. Agrociencia 49(4):423438.

UNALM (Universidad Nacional Agraria La Molina). 2018. UNALM: Jardín Botánico-Clima del distrito La Molina (en línea, sitio web). Consultado 10 mar. 2018. Disponible en http://www.lamolina.edu.pe/facultad/ciencias/ cbiologia/jardin botanico/Ubicacion.html.

Presentado: $18 / 05 / 2018$ Aceptado: 29/06/2018 